\title{
VIEWPOINT
}

\section{Rethinking the Standard of Care for Myalgic Encephalomyelitis/Chronic Fatigue Syndrome}

\author{
Fred Friedberg, $P h . D^{7}$ (D) Madison Sunnquist, $M A^{2}$, and Luis Nacul, MD, Ph. $D^{3,4}$ \\ ${ }^{1}$ Stony Brook University, Stony Brook, NY, USA; '2DePaul University, Chicago, IL, USA; ${ }^{3}$ London School of Hygiene \& Tropical Medicine, London, UK; \\ ${ }^{4}$ Complex Chronic Diseases Program, British Columbia Women's Hospital, Vancouver, Canada.
}

J Gen Intern Med 35(3):906-9

DOI: $10.1007 /$ s11606-019-05375-y

(C) Society of General Internal Medicine 2019

$\mathrm{F}$ or over two decades, the standard of care for myalgic encephalomyelitis/chronic fatigue syndrome (ME/CFS) has been cognitive behavior therapy (CBT) and graded exercise therapy (GET). Both interventions had been recommended by the US Centers for Disease Control and the UK NICE guidelines. ${ }^{1}$ Behavioral intervention as the clinical standard was given a considerable boost by the 5 million-pound PACE trial, a large multi-arm randomized trial of CBT and GET launched in $2007 .^{2}$ This British government-funded trial was intended to definitively answer whether such interventions were beneficial in ME/CFS. In their 2011 and 2013 publications, the PACE trial authors announced with widespread publicity that $22 \%$ of their patients had "recovered" and 59$61 \%$ had clinically improved across the CBT and GET interventions. $^{2,3}$

More generally, multiple literature reviews have reported that these therapies are not only effective at improving fatigue and, to a lesser extent, physical function in ME/CFS but also safe. ${ }^{4-6}$ It would seem obvious then that good clinical care of these patients would include these behavioral interventions. But, a closer look at these trials has generated many concerns about their applicability to these patients. This perspective critically examines their findings and more generally discusses the behavioral intervention literature in ME/CFS. Finally, we briefly describe a pragmatic clinical approach for these oftenmarginalized patients.

\section{CLAIMS OF RECOVERY AND CLINICAL IMPROVEMENT}

The long-running controversy over recovery and improvement claims in ME/CFS clinical trials may be unique to this stigmatized illness. ${ }^{7}$ Substantial recovery from ME/CFS is of

Received May 18, 2019

Revised August 7, 2019

Accepted September 12, 2019

Published online October 21, 2019 course desirable but quite uncommon. A 2005 systematic review ${ }^{8}$ reported that a median of only $5 \%$ of these patients regained full health. It was therefore surprising when the flagship PACE trial reported that a majority of patients clinically improved and about $1 / 5$ recovered following CBT and GET. $^{2,3}$ These widely publicized findings have had a significant influence on governmental agencies, healthcare institutions, and clinical guidelines which have promoted CBT and GET as the standard of care for ME/CFS, e.g., Centers for Disease Control and Prevention. ${ }^{1}$

These behavioral intervention studies, grounded in psychological models of causation and cure, ${ }^{9}$ have probably impacted biomedical research as well. Consider the low funding rates of $\mathrm{ME} / \mathrm{CFS}$ research at the National Institutes of Health (averaging \$5-6 M/year), often in the bottom 10 of over 200 conditions tracked in the last two decades. ${ }^{10}$ This has improved recently with funding of three collaborative research centers but is still substantially below what would be commensurate with disease burden. ${ }^{11}$

Since its publication, the PACE trial has been widely criticized for a number of study design issues, including (i) use of a recovery definition that did not require restoration of health, e.g., Adamowicz et al., ${ }^{12}$ and (ii) loosening of clinical outcome criteria during the trial ${ }^{13,14}$ that dramatically inflated its success. For instance, changes to recovery criteria resulted in $13 \%$ of patients meeting an important recovery criterion (physical functioning) at baseline before the trial started. ${ }^{15}$. Formal reanalysis of the PACE trial data ${ }^{14}$ using original outcome criteria, ${ }^{13}$ rather than the far looser mid-trial revised criteria,

3 found that clinical improvement for CBT and GET dropped from 59-61 to $20-21 \%$ and recovery levels dropped from 22 to $4-7 \%$. This is similar to the $3 \%$ recovery rate in their medical care controls and the low recovery rates found in Cairns and Hotopf's ${ }^{8}$ literature review.

\section{NON-BLINDED TRIALS AND SUBJECTIVE MEASURES}

In general, studies of CBT and GET in this illness have not been blinded and relied on subjective outcome measures, which is common in behavioral intervention trials. However, given the important influence of the PACE trial on the standard of care, the potential biasing effects of subjective measures have been more closely examined. ${ }^{16}$ Overall, objective 
outcomes ${ }^{17}$ did not support PACE efficacy claims based on self-report measures. Of the four objective measures in the PACE trial, i.e., 6-min walk test, fitness step test, days lost from work, ${ }^{18}$ and percent on disability benefits, none were found to show a selective benefit for CBT or GET. In addition, the 6-min walk test, a brief measure of functional capacity, yielded post-treatment data that fell far short of normative values that would suggest recovery. ${ }^{15,}{ }^{19}$ Finally, no verified reports of return to work were published, another important indicator of recovery.

The lack of objective improvement in the PACE trial is consistent with three CBT trials in ME/CFS that found improved self-report outcomes, but no significant improvement in objective activity levels. ${ }^{20}$ This is contrary to the widely cited behavioral model of chronic fatigue syndrome ${ }^{21}$ that predicts increased physical functioning as a result of graded exercise. Alternatively, it has been suggested that patientreported improvements on subjective measures may reflect improved coping, stress reduction, or improved adjustment to ongoing limitations, ${ }^{22}$ rather than robust gains in physical and role functioning.

\section{OVERLY BROAD ILLNESS CRITERIA}

The most commonly used research criteria defining chronic fatigue syndrome are based on the 1994 Centers for Disease Control ("Fukuda") case definition. ${ }^{23}$ However, the PACE trial and many other CBT and GET studies in ME/CFS used the broadest definition of the illness, i.e., Oxford criteria, ${ }^{24}$ which requires only chronic fatigue rather than the typical multi-symptom presentation of non-restorative sleep, cognitive difficulties, and post-exertional malaise (sustained postexertion worsening of symptoms). ${ }^{25}$ These additional symptoms identify a more debilitated group in comparison to chronic fatigue alone which may be more responsive to behavioral intervention. ${ }^{26}$ Notably, the use of Oxford criteria has predominated in literature reviews (e.g., Price et al. ${ }^{4}$ and Larun et al. ${ }^{6}$ ) where significant minorities of treated participants reported positive effects of behavioral intervention.

The 2014 review ${ }^{27}$ of CBT and GET studies sponsored by the US Agency for Health Care Research and Quality (AHRQ) reported moderate effect sizes for both therapies, based in large part on Oxford-defined illness. But, that review also concluded that the Oxford definition could include "patients who may have an alternate fatiguing illness [i.e. other than ME/CFS]." When AHRQ excluded Oxford-based studies from its analysis, leaving only Fukuda-defined chronic fatigue syndrome ${ }^{23}$ studies, it found insufficient evidence of beneficial effect for GET and barely any for $\mathrm{CBT}^{27}$ The AHRQ report also noted that studies requiring the core symptom of post-exertional malaise were "blatantly missing" from the evidence base. In its 2015 report ${ }^{28}$ the National Institutes of Health concluded that the use of Oxford-defined illness could "impair progress and cause harm" and recommended it be retired.

\section{FLAWED DISEASE MODEL}

The cognitive behavioral model of chronic fatigue syndrome posits that the symptoms and debility of ME/CFS are the result of deconditioning, originating from patients' fear of activity and false cognitions that the illness is physical. ${ }^{16,19,20}$ As stated in the PACE trial manual:" "According to this model, the symptoms and disability of ME/CFS are perpetuated ... by unhelpful [fear-based] illness beliefs and [avoidant] coping behaviours" (p. 18). Thus, CBT interventions for chronic fatigue syndrome focus on changing illness beliefs (cognitions) to promote increased activity (behavior). The GET program was designed to help ME/CFS patients overcome this purported fear of exercise through graded exposure to exercise, which would also reverse deconditioning. ${ }^{9}$ Both of these treatments suggest that increasing activity will alleviate symptoms and restore health. The re-analysis of the PACE trial poses major challenges to the cognitive behavioral model.

One unfortunate consequence of the model is that $\mathrm{ME} / \mathrm{CFS}$ has come to be viewed, not as a biologically based illness, but rather as a psychological ailment that is reversible with behavioral therapies. ${ }^{29}, 30$ This model stands in stark contrast to the harsh reality of this disabling condition and the significant evidence of neurological, immunological, autonomic, and energy metabolism impairment, as reviewed in an influential 2015 Institute of Medicine report. ${ }^{31}$ The appropriate use of CBT, which may be selectively indicated in a number of chronic diseases, such as cancer, is intended to promote patients' ability to cope with their illnesses in order to improve quality of life, e.g., Daniels. ${ }^{32}$ CBT is not viewed as a cure for these illnesses, nor is it used to convince patients that they are either psychologically ill or simply not ill as has been done in ME/CFS, cf. Geraghty et al. ${ }^{29}$ Thus, the use of CBT as a curative intervention for chronic fatigue syndrome deviates from the treatment's intended purpose.

\section{EVIDENCE OF HARMS}

Literature reviews have often reported that CBT and GET for patients with ME/CFS are safe. ${ }^{6,}{ }^{33}$ But, claims of safety are not adequately supported by the evidence and are contradicted by the experiences of clinicians and patients. The 2014 AHRQ review ${ }^{27}$ reported, "Harms [worsening of symptoms and/or disability] were generally inadequately reported across trials" (p. vi). When harms were reported, the AHRQ report concluded that GET studies reported more adverse events and withdrawals. Experienced clinicians also do not recommend PACE-type GET or CBT as treatments. ${ }^{34}$

Furthermore, in an analysis of large international patient surveys, ${ }^{35}$ over $50 \%$ of patients reported that CBT and GET not only fell short of delivering significant improvements but 
often led to worsened health due to ill-advised activity and exercise prescriptions. The surprising lack of attention to these patient-reported negative outcomes appears to be the result of skepticism of $\mathrm{ME} / \mathrm{CFS}^{7}$ and the prioritizing of results from clinical trials without considering important inputs from seasoned practitioners and patients.

\section{CARING FOR ME/CFS PATIENTS}

In clinical practice, many individuals presenting with the common symptom of persistent fatigue may benefit from activity-based behavioral interventions, e.g., Friedberg et al. $^{26}$ However, persistent fatigue is not equivalent to the multi-symptom debilitating illness of ME/CFS. Despite the lack of approved treatments or a fully articulated standard of medical care, there are still many actions physicians can take to help these underserved patients. First, practitioners can acknowledge the biomedical reality of the illness and their belief that the patient is genuinely ill. Next, clinicians can help patients to better manage a major illness challenge: how to minimize debilitating post-exertional malaise by learning to stay within their "energy envelope." 36

The energy envelope delineates the amount of energy that a ME/CFS patient has available to perform all activities. ${ }^{37}$ The size of this energy envelope can vary from day to day and between patients with some patients lacking energy for basic activities of daily living. When patients exceed their limited energy levels, they experience post-exertional worsening of symptoms and functioning. Medical providers can teach patients how to recognize their own personal energy limits and use "pacing" (dividing symptom-producing activities into smaller parts with interspersed rest intervals) to stay within those limits. ${ }^{34,37}$ Once pacing is effectively used, some patients may be able to use an individualized exercise plan to increase available energy and functioning while avoiding postexertional worsening. ${ }^{34,} 36$

Practitioners can also help patients with appropriate pharmacological and non-pharmacological treatments. ${ }^{38,39}$ This includes treatments for unrefreshing sleep, e.g., trazodone and low-dose tricyclic antidepressants, and sleep hygiene measures. In addition, pain can be addressed with low-dose naltrexone $^{40}$ and anti-epileptics, e.g., gabapentin, and orthostatic intolerance can be treated with fludrocortisone and salt loading. Comorbidities can be managed using standard of care. Drugs should usually be started at low doses because patients can be sensitive to medications. If needed, patients can be referred to counseling to improve coping with the severe impacts of ME/CFS on quality of life.

For optimal patient care, we recommend a ME/CFS specialist or a specialist center supported by a multi-disciplinary team. Unfortunately, few of these practitioners or centers are available, which highlights the need for provider education and training regarding this illness. Realistically, when specialists are not available, care is best provided by the generalist (internal medicine or family doctor) working as part of a multidisciplinary team including expertise (as available) in immunology, infectious disease, cardiology or neurology, psychology, occupational therapy, and social work. With this interprofessional approach, practitioners can lessen harms while helping patients improve their health, function, and quality of life to the extent possible. Further information on clinical management may be found in the following sources: a free practitioner's guide to $\mathrm{ME} / \mathrm{CFS},{ }^{34}$ a clinically focused review, $^{41}$ and a pragmatic clinical paper. ${ }^{36}$

Acknowledgments: We thank Mary Dimmock for her thoughtful input on this paper.

Corresponding Author: Fred Friedberg, Ph.D; Stony Brook University, Stony Brook, NY, USA (e-mail: fred. friedberg@stonybrookmedicine.edu).

\section{Compliance with Ethical Standards:}

Conflict of Interest: The authors declare that they do not have a conflict of interest.

\section{REFERENCES}

1. Centers for Disease Control and Prevention. Management of Chronic Fatigue Syndrome (CFS). Website as of May 17, 2017. Last accessed on August 2, 2019.

2. White PD, Goldsmith KA, Johnson AL, Potts L, Walwyn R, DeCesare JC, Baber HL, Burgess M, Clark LV, Cox DL, Bavinton J, Angus BJ, Murphy G, Murphy M, O'Dowd H, Wilks D, McCrone P, Chalder T, Sharpe M. Comparison of adaptive pacing therapy, cognitive behaviour therapy, graded exercise therapy, and specialist medical care for chronic fatigue syndrome (PACE): a randomized trial. Lancet. 2011;377(9768): 823-836.

3. White PD, Goldsmith K, Johnson AL, Chalder T, Sharpe M. PACE Trial Management Group. Recovery from chronic fatigue syndrome after treatments given in the PACE trial. Psychol Med. 2013;43(10): 22262235. https://doi.org/10.1017/S0033291713000020.

4. Price JR, Mitchell E, Tidy E, Hunot V. Cognitive behaviour therapy for chronic fatigue syndrome in adults. Cochrane Database Syst Rev. 2008;3:CD001027.

5. Malouff JM, Thorsteinsson EB, Rooke SE, Bhullar N, Schutte NS. Efficacy of cognitive behavioral therapy for chronic fatigue syndrome: a meta-analysis. Clin Psychol Rev. 2008; 28(5), 736-745.

6. Larun L, Brurberg KG, Odgaard-Jensen J, Price JR. Exercise therapy for chronic fatigue syndrome. Cochrane Database Syst Rev. 2017. http:// onlinelibrary.wiley.com/doi/10.1002/14651858.CD003200.pub7/full.

7. Blease C, Carel H, Geraghty $\mathbf{K}$. Epistemic injustice in healthcare encounters: evidence from chronic fatigue syndrome. J Med Ethics. 2017;43(8)549-557.

8. Cairns R, Hotopf M. A systematic review describing the prognosis of chronic fatigue syndrome. Occup Med. 2005;55(1), 20-31.

9. Burgess M, Chalder T. Manual for therapists: cognitive behavior therapy for CFS/Me. PACE Trial Management Group: CBT Therapists Manual. 2004 .

10. National Institutes of Health. NIH research portfolio online reporting tools. Project information. 2014.

11. Dimmock ME, Mirin AA, Jason LA. Estimating the Disease Burden of $\mathrm{ME} / \mathrm{CFS}$ in the United States and Its Relation to Research Funding. J Med Ther. 2016;1(1).

12. Adamowicz JL, Caikauskaite I, Friedberg F, Seva V. Patient change attributions in self-management of severe chronic fatigue syndrome. Fatigue: Biomedicine, Health \& Behavior. 2017; 5(1), 21-32.

13. White PD, Sharpe MC, Chalder T, DeCesare JC, Walwyn R. Protocol for the PACE trial: A randomised controlled trial of adaptive pacing, cognitive behaviour therapy, and graded exercise as supplements to standardised specialist medical care versus standardized specialist medical care alone for patients with the chronic fatigue syndrome/myalgic encephalomyelitis or encephalopathy. BMC Neurol. 2007;7(1)6. 
14. Wilshire CE, Kindlon T, Courtney R, Matthees A, Tuller D, Geraghty $\mathbf{K}$, Levin B. Rethinking the treatment of chronic fatigue syndrome-a reanalysis and evaluation of findings from a recent major trial of graded exercise and CBT. BMC Psychol.. 2018;6(1):6.

15. Wilshire C, Kindlon T, Matthees A, McGrath S. Can patients with chronic fatigue syndrome really recover after graded exercise or cognitive behavioural therapy? A critical commentary and preliminary re-analysis of the PACE trial. Fatigue: Biomedicine, Health \& Behavior. 2016. http:// www.tandfonline.com/doi/full/10.1080/21641846.2017.1259724.

16. Lubet $\mathbf{S}$. Investigator bias and the PACE trial. J Health Psychol. 2017;22(9)1123-1127.

17. McPhee G. Cognitive behaviour therapy and objective assessments in chronic fatigue syndrome. J Health Psychol. 2017;22(9)1181-1186.

18. McCrone $\mathbf{P}$, Sharpe M, Chalder T, Knapp M, Johnson AL, Goldsmith KA, White PD. Adaptive pacing, cognitive behaviour therapy, graded exercise, and specialist medical care for chronic fatigue syndrome: a costeffectiveness analysis. PLoS One. 2012;7(8), e40808.

19. Agardy S. Chronic fatigue syndrome patients have no reason to accept the PACE trial results: Response to Keith J Petrie and John Weinman. J Health Psychol. 2017; 22(9), 1206-1208.

20. Wiborg JF, Knoop H, Stulemeijer M, Prins JB, Bleijenberg G. How does cognitive behaviour therapy reduce fatigue in patients with chronic fatigue syndrome? The role of physical activity. Psychol Med. 2010;40(8), 1281-1287.

21. Wessely S, Butler S, Chalder T, David A. The cognitive behavioural management of the post-viral fatigue syndrome. In Post-viral fatigue syndrome. 1991; (pp. 305-334). John Wiley \& Sons Ltd.

22. Whiting P, Bagnall AM, Sowden AJ, Cornell JE, Mulrow CD, Ramirez G. (2001). Interventions for the treatment and management of chronic fatigue syndrome: A systematic review. JAMA.;286:1378-1379.

23. Fukuda K, Straus SE, Hickie L, Sharpe MC, Dobbins JG, Komaroff A. The chronic fatigue syndrome: A comprehensive approach to its definition and study. Ann Intern Med. 1994;121:953-959.

24. Sharpe MC; Archard LC; Banatvala JE; et al. A report-chronic fatigue syndrome: guidelines for research. J R Soc Med. 1991;84(2):118-21. doi:https://doi.org/10.1177/014107689108400224.

25. Jason LA, Sunnquist M, Abigail B, Evans M, Vernon SD, Furst J, Simonis V. Examining case definition criteria for chronic fatigue syndrome and myalgic encephalomyelitis. Fatigue. 2014;2(1):40-56. Published online 2013 Dec 11. https://doi.org/10.1080/21641846.2013.862993.

26. Friedberg F, Napoli A, Coronel J, Adamowicz J, Seva V, Caikauskaite I, Ngan M, Chang $\mathbf{J}$, Meng $\mathbf{H}$. Chronic fatigue self-management in primary care. A randomized trial. Psychosom Med. 2013;75(7):650-7. https://doi.org/10.1097/PSY.0b013e31829dbed4.

27. Agency for Healthcare Research and Quality. Diagnosis and Treatment of Myalgic Encephalomyelitis/Chronic Fatigue Syndrome. Evidence Report/ Technology Assessment Number 219. AHRQ Publication No. 15-E001-EF U.S. Department of Health and Human Services; 2014.

28. Green CR, Cowan P, Elk R, O'Neil KM, Rasmussen AL. National Institutes of Health pathways to prevention workshop: advancing the research on myalgic encephalomyelitis/chronic fatigue syndrome. Ann Intern Med. 2015;162(12)860-865.

29. Geraghty KJ, Blease C. Myalgic encephalomyelitis/chronic fatigue syndrome and the biopsychosocial model: a review of patient harm and distress in the medical encounter. Disabil Rehabil. 2018;1-10.
30. Geraghty K, Jason L, Sunnquist M, Tuller D, Blease C, Adeniji C. The 'cognitive behavioural model' of chronic fatigue syndrome: Critique of a flawed model. Health Psychol Open. 2019;6(1), 2055102919838907.

31. Beyond Myalgic Encephalomyelitis/Chronic Fatigue Syndrome: Redefining an Illness. Committee on the Diagnostic Criteria for Myalgic Encephalomyelitis/Chronic Fatigue Syndrome; Board on the Health of Select Populations; Institute of Medicine. Washington (DC): National Academies Press (US); 2015

32. Daniels S. Cognitive behavior therapy for patients with cancer. J Adv Pract Oncol. 2015;6(1)54.

33. Reid S, Chalder T, Cleare A, Hotopf $\mathbf{M}$, Wessely S. Chronic fatigue syndrome. Clin Evid. 2011.

34. Friedberg F, Bateman L, Bested AC, Friedman KJ, Gurwitt A, Lapp CW, Vallings R. ME/CFS: A primer for clinical practitioners. Chicago: International Association for Chronic Fatigue Syndrome/Myalgic Encephalomyelitis. 2012.

35. Kindlon T. Reporting of harms associated with graded exercise therapy and cognitive behavioural therapy in myalgic encephalomyelitis/chronic fatigue syndrome. Bull the IACFS/ME. 2011;19(2)59-111. Myalgic Encephalomyelitis/Chronic Fatigue Syndrome: Redefining an Illness. Washington (DC): National Academies Press (US); 2015 Feb.

36. Goudsmit EM, Nijs J, Jason LA, Wallman KE. Pacing as a strategy to improve energy management in myalgic encephalomyelitis/chronic fatigue syndrome: a consensus document. Disabil Rehab. 2012;34(13)1140-1147.

37. Jason L, Brown M, Brown A, Evans M, Flores S, Grant-Holler E, Sunnquist M. Energy Conservation/Envelope Theory Interventions. Fatigue: Biomedicine, Health \& Behavior 1, no. 1-2 (April 1, 2013): 27-42. https://doi.org/10.1080/21641846.2012. 733602 .

38. Carruthers BM, Jain AK, De Meirleir KL, Peterson DL, Klimas NG, Lerner AM, Bested AC, Flor-Henry P, Joshi P, Powles ACP, Sherkey JA, van de Sande MI. Myalgic Encephalomyelitis/ Chronic Fatigue Syndrome. Clinical Working Case Definition, Diagnostic and Treatment Protocols. Journal of Chronic Fatigue Syndrome, 2003;11:(1)7-115. https://doi.org/10.1300/ J092v11n01_02.

39. Lapp C, Front W. Pediatr., 23 January 2019. Initiating Care of a Patient With Myalgic Encephalomyelitis/Chronic Fatigue Syndrome (ME/CFS). https://doi.org/10.3389/fped.2018.00415.

40. Tucker M. Low-Dose Naltrexone Explored as Option for Chronic Pain. Medscape. 2018. http://www.medscape.com/viewarticle/894020. Accessed 2 Aug 2019.

41. Bested A, Marshall L. Review of Myalgic Encephalomyelitis/ Chronic Fatigue Syndrome: An Evidence-Based Approach to Diagnosis and Management by Clinicians. Rev Environ Health. (2015);30(4)223-49. https://doi.org/10.1515/reveh-20150026 .

Publisher's Note Springer Nature remains neutral with regard to jurisdictional claims in published maps and institutional affiliations. 